\title{
OPEN
}

Published online: 26 February 2020

\section{Author Correction: The beneficial effects of Lactobacillus reuteri ADR-1 or ADR-3 consumption on type 2 diabetes mellitus: a randomized, double-blinded, placebo-controlled trial}

\author{
Ming-Chia Hsieh, Wan-Hua Tsai, Yu-Pang Jheng, Shih-Li Su, Shu-Yi Wang, Chi-Chen Lin, \\ Yi-Hsing Chen \& Wen-Wei Chang (1)
}

Correction to: Scientific Reports https://doi.org/10.1038/s41598-018-35014-1, published online 14 November 2018

This Article contains errors in the Materials and Methods and the Results section.

In the Materials and Methods section under subheading 'The recruitment of participants',

"The exclusion criteria were as follows: pregnancy; the presence of other diseases, including cancers (with the exception of well-controlled benign tumors), kidney failure/dialysis, heart diseases, stroke, or autoimmune diseases; the use of medications, including anti-diabetes drugs, and antibiotics, or other probiotic products $<4$ weeks before randomization; an AST/ALT level $>3$-fold the normal range; participation in other clinical trials; and the presence of other medical conditions that might jeopardize compliance with the protocol (e.g., malabsorption syndrome or an inability to take orally administered drugs)."

should read:

"The exclusion criteria were as follows: pregnancy; the presence of other diseases, including cancers (with the exception of well-controlled benign tumors), kidney failure/dialysis, heart diseases, stroke, or autoimmune diseases; the use of medications, including anti-diabetes herbs, and antibiotics, or dietary supplements for diabetes care as well as other probiotic products $<4$ weeks before randomization; an AST/ALT level $>3$-fold the normal range; participation in other clinical trials; and the presence of other medical conditions that might jeopardize compliance with the protocol (e.g., malabsorption syndrome or an inability to take orally administered drugs)."

In the Results section under subheading 'L. reuteri strain ADR-1 displayed beneficial effects in a T2DM rat model'.

"Reductions in serum antioxidant proteins such as glutathione reductase (GPX) and superoxide dismutase (SOD) were also observed in HFD rats with oral administration of ADR-1 (Fig. 1D).”

should read:

Recovery of down-regulated expression in glutathione reductase (GPX) and superoxide dismutase (SOD) was observed in HFD rats with oral administration of ADR-1 (Fig. 1D). 
(c) (i) Open Access This article is licensed under a Creative Commons Attribution 4.0 International License, which permits use, sharing, adaptation, distribution and reproduction in any medium or format, as long as you give appropriate credit to the original author(s) and the source, provide a link to the Creative Commons license, and indicate if changes were made. The images or other third party material in this article are included in the article's Creative Commons license, unless indicated otherwise in a credit line to the material. If material is not included in the article's Creative Commons license and your intended use is not permitted by statutory regulation or exceeds the permitted use, you will need to obtain permission directly from the copyright holder. To view a copy of this license, visit http://creativecommons.org/licenses/by/4.0/.

(C) The Author(s) 2020 